\title{
General Impedance Synthesis Using Simple Switching Converters
}

\author{
Joe C. P. Liu* ${ }^{* \dagger}$, Chi K. Tse*, Franki N. K. Poon ${ }^{\dagger}$, Bryan M. H. Pong ${ }^{\ddagger}$ and Y. M. Lai* \\ * Department of Electronic and Information Engineering, Hong Kong Polytechnic University, Hong Kong \\ ${ }^{\dagger}$ PowerELab Limited, Technology Innovation and Incubation Building, University of Hong Kong, Hong Kong

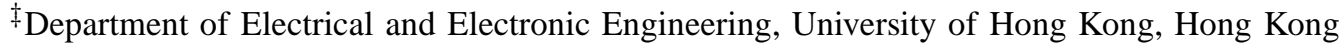

\begin{abstract}
A general impedance synthesizer using a minimum number of switching converters is studied in this paper. We begin with showing that any impedance can be synthesized by a circuit consisting of only two simple power converters, one storage element (e.g., capacitor), and one dissipative element (e.g., resistor) or power source. The implementation of such a circuit for synthesizing any desired impedance can be performed by (i) programming the input current given the input voltage such that the desired impedance function is achieved; (ii) controlling the amount of power dissipation (generation) in the dissipative element (source) so as to match the required active power of the impedance to be synthesized. Then, the instantaneous power will automatically be balanced by the storage element. Such impedance synthesizers find a lot of applications in power electronics. For instance, a resistance synthesizer can be used for power factor correction (PFC), a programmable capacitor or inductor synthesizer (comprising of small high-frequency converters) can be used for control applications.
\end{abstract}

\section{INTRODUCTION}

Many problems in electrical engineering are reducible to one of impedance synthesis or imitation [1]-[3]. Basically, the impedance observed from the terminals of a given circuit is defined as the ratio of the voltage across the terminals and the current flowing into and out of the terminals, as shown in Fig. 1. In power electronics, for instance, impedance imitation is central to many applications, be it known or clearly recognized by the engineers. Power factor correction (PFC), for example, effectively requires the input impedance to be resistive [4], [5]. Thus, imitating a resistor for the input of a converter is the basic requirement for achieving a high power factor for the converter. Many control problems can also be interpreted as a kind of impedance modification, e.g., shaping the load transient of a converter is essentially a process of modifying the output impedance of the converter [7]. In this paper, we consider the problem of synthesizing impedance using a minimal configuration of switching converters. The problem is relevant to power electronics, and may also be applicable to other branches of electrical engineering. For instance, programmable reactive components using some highfrequency converters are useful elements for control applications.

We will begin with a review of switching converters in Section II, with particular emphasis on their fundamental functions in terms of terminal voltages and currents. The main task, which will be presented in Section III, is to find the minimal configuration for synthesizing any impedance.

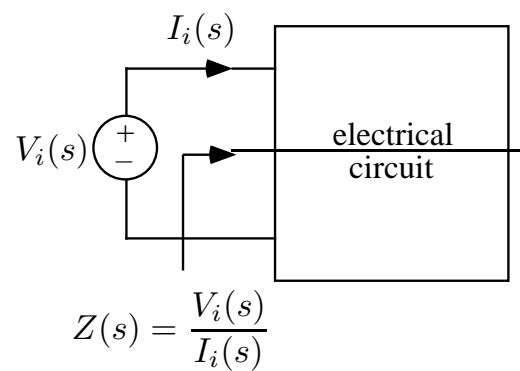

Fig. 1. Impedance definition. $Z(s)$ is the impedance observed at the input terminals of the given electrical circuit.

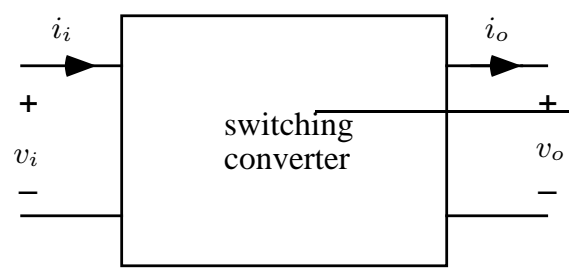

Fig. 2. Simple switching converter as two-port.

Then, in Section IV, we will present a design of such an impedance synthesizer, and provide some experimental results in Section V.

\section{REVIEW OF SWITCHING CONVERTERS}

We consider a simple switching converter as a two-port circuit, as shown in Fig. 2. Let the input voltage, input current, output voltage and output current be $v_{i}, i_{i}, v_{o}$ and $i_{o}$, respectively. In practice, a simple switching converter may contain a high-frequency storage element and a pair of switches, such as the buck, buck-boost and boost converters, as shown in Fig. 3 [6]. Note that the output capacitor is not considered as part of the converter and the converter therefore has no low-frequency storage capability. The switches are switched periodically at a high frequency (usually hundreds of $\mathrm{kHz}$ ). The switching frequency can be regarded as being so high that all variables $v_{i}, i_{i}, v_{o}$ and $i_{o}$ are relatively slowly varying. ${ }^{1}$ Precisely, we write

$$
f_{\max } \ll f_{s}
$$

\footnotetext{
${ }^{1}$ This assumption holds for all power electronics applications. Ideal switching converters therefore operate at infinite switching frequency.
} 


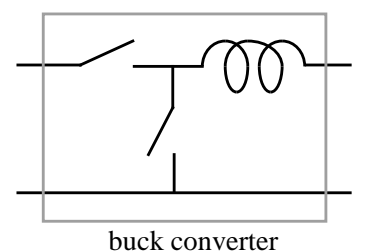

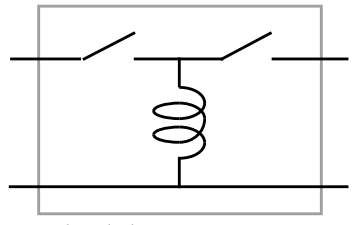

buck-boost converter

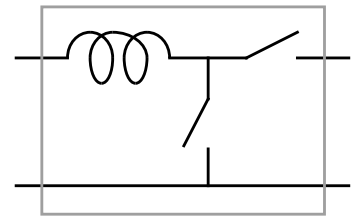

boost converter
Fig. 3. Simple switching converters for voltage source terminations. Duals of these circuits can be likewise derived for current source terminations using capacitor and switches.

where $f_{\max }$ is the maximum frequency of $v_{i}, i_{i}, v_{o}$ and $i_{o}$, and $f_{s}$ is the switching frequency of the converter. Specific power processing functions are achieved by adjusting or controlling the relative durations of the "on" and "off" intervals of the switches.

The most fundamental property of an ideal switching converter is the conservation of power under slowly varying terminal voltage and current conditions. Clearly, the average input power over one repetition period (also called switching period) must equal the average output power over the same period, i.e., $\int_{T} v_{i} i_{i} d t=\int_{T} v_{o} i_{o} d t$. Here we emphasize that the equality of energy is valid only if the switching frequency is very much higher than the variation of all terminal variables. Thus, the ideal converter does not store or dissipate any energy over a repetition period under the condition of slowly varying terminal voltages and currents. Of course, real converters are never $100 \%$ efficient, but here, we ignore this loss to keep our discussion simple. Moreover, in practice, we only consider $v_{i}, i_{i}, v_{o}$ and $i_{o}$ being slowly varying, i.e., varying at a frequency which is much lower than the switching frequency of the converter. Hence, the ideal power conservation equation becomes

$$
v_{i} i_{i}=v_{o} i_{o}, \quad \text { (“ } \approx \text { " in practice) }
$$

where $v_{i}, i_{i}, v_{o}$ and $i_{o}$ can be regarded as "instantaneous" variables if they satisfy (1). In other words, equation (2) holds only when we consider low-frequency (i.e., slowly varying) voltage and current variables. Precisely, in practice, (2) is an approximation, the integrity of which improves as the switching frequency gets higher. ${ }^{2}$

In addition to the power conservation property, a defining objective of a switching converter is that given one of the terminal variables (normally the input voltage $v_{i}$ ) and possibly some constraints on the terminal variables (as, for instance, enforced by the load characteristics), a switching converter

\footnotetext{
${ }^{2}$ For any of the simple converters, viz. buck, boost and buck-boost converters shown in Fig. 3 where low-frequency storage is absent, the inductor stores negligible energy over one switching period under slowly varying terminal voltage and current conditions.
}

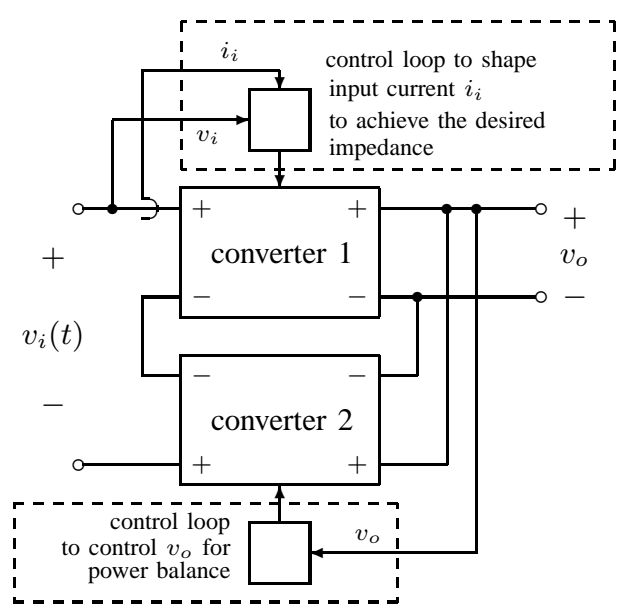

Fig. 4. A minimal configuration, with at least one low-frequency storage within either converter 1 or 2 .

attempts to regulate or program one or more of the remaining three terminal variables by controlling some parameter. For example, for dc power supplies, the input voltage is given, and the switching converter aims to regulate the output voltage by controlling the duty cycle. Moreover, for power factor correction, the input voltage is given (normally a rectified sinewave), and the switching converter aims to program the input current such that it varies at the same frequency and in phase with the input sinewave voltage.

Note that the above definition implicitly assumes that all terminal voltages and currents are slowly varying compared to the switching frequency. Thus, theoretically, if the switching frequency approaches infinity, the switching converter can process voltage or current of arbitrarily high frequencies.

\section{Minimal Configuration of Impedance Synthesizer BASED ON SWITCHING CONVERTERS}

In finding the basic configuration of an impedance synthesizer, we first observe that an impedance can be realized by programming the current if the voltage is given, and vice versa. This fits the definition of the switching converter described in Section II. Thus, a switching converter can be used to program the input current $i_{i}$ given its input voltage $v_{i}$ in order to create the desired impedance seen from the converter's input. ${ }^{3}$ Here, both $v_{i}$ and $i_{i}$ are slowly varying in the sense of (1). Moreover, the switching converter is subject to the constraint of (2), i.e., power conservation. Clearly, general impedance cannot be synthesized with one converter terminated with a fixed load impedance because (2) cannot generally be satisfied. ${ }^{4}$ Thus, one switching converter is insufficient for general impedance synthesis. Clearly, we need to balance the power by ensuring that the output of the converter "emits" the right amount of "instantaneous" power. Note again that "instantaneous" refers

\footnotetext{
${ }^{3}$ This is exactly what a PFC converter does.

${ }^{4} \mathrm{~A}$ single converter may be able to synthesize a particular type of impedance. For instance, a resistive input impedance ( $\mathrm{PFC}$ converter) can be constructed out of one converter with a mandatory dissipative load and a low-frequency storage capacitor.
} 


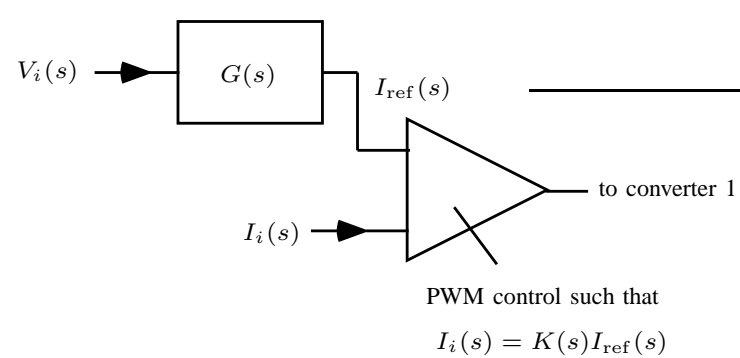

Fig. 5. Current shaping control for achieving the required magnitude and phase relationships between input voltage and input current. $K(s)$ is nearly constant if all variables are slowly varying relative to the switching frequency.

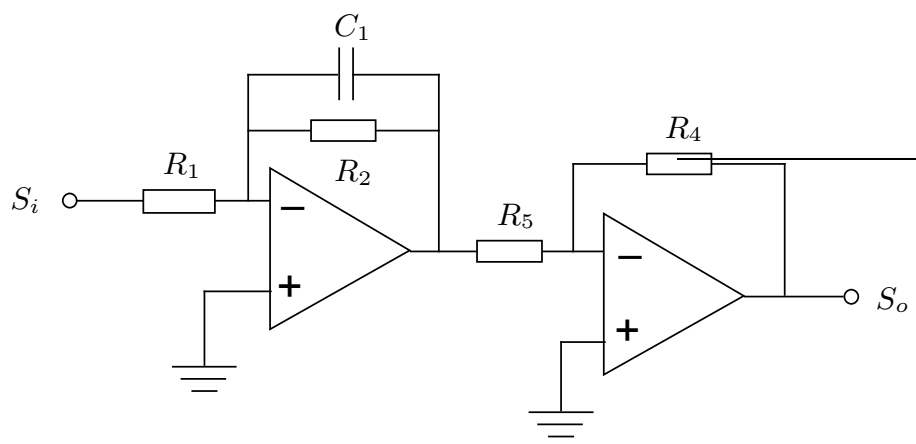

Fig. 6. Circuit implementation of $G(s)$ for inductive impedance synthesis. Here, $G(s)=\frac{S_{o}}{S_{i}}=1 /\left[\frac{R_{1}}{R_{2}}+s C_{1} R_{1}\right]$, with $R_{4}=R_{5}$.

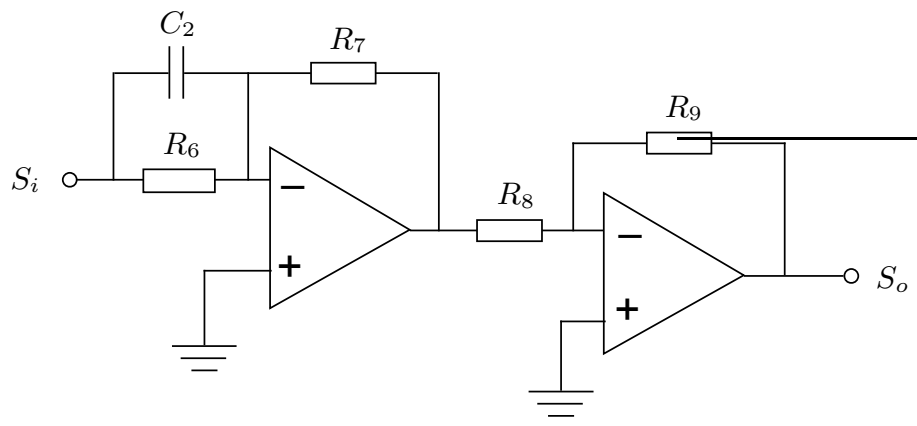

Fig. 7. Circuit implementation of $G(s)$ for capacitive impedance synthesis. Here, $G(s)=\frac{S_{0}}{S_{i}}=\frac{R_{7}}{R_{8}}\left[1+s C_{2} R_{6}\right]$, with $R_{8}=R_{9}$.

to relatively low-frequency variables assuming a much higher switching frequency.

If two switching converters are available, one of them can be used to program the input current (given the input voltage) so as to achieve the desired impedance. The other can then be used to match the power conservation requirement by controlling its output to dissipate or generate the correct amount of real power since $v_{i} i_{i}=v_{o} i_{o}$ must be satisfied by both converters. This is possible according to the definition of the switching converter described in Section II that, given the load (a resistor or a dc current source), the converter can adjust/control its output voltage $v_{o}$ so as to emit or absorb a desired amount of dc power to or from the output port. Clearly, there must then exist a storage element connecting

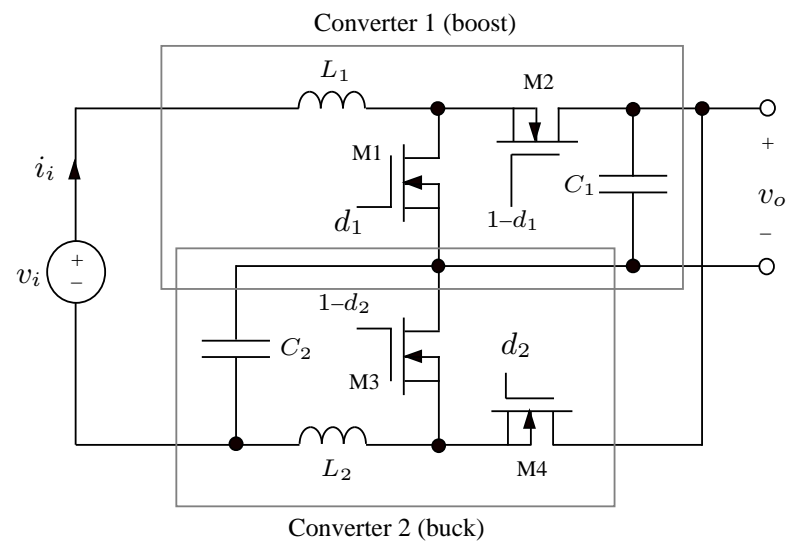

Fig. 8. Experimental impedance synthesizer based on the configuration of Fig. 4, with converter 1 and converter 2 realized by a boost converter and a buck converter, respectively. $C_{2}$ serves as internal storage.

between the two converters in order to absorb the right amount of instantaneous power to meet the power balance for both converters. Thus, the minimum configuration consists of

- two switching converters,

- one storage element (at frequencies up to $f_{\max } \ll f_{s}$ ),

- one dissipative element or power source.

Obviously the dissipative element can be realized by a negative power source (e.g., current load). Furthermore, because power flow can be in either directions, the converters must be bidirectional.

\section{IMPLEMENTATION}

Some suitable circuit configurations satisfying the above conditions can be found in Liu et al. [8], which describes a family of two-converter configurations for achieving two control functions. A minimal configuration is shown in Fig. 4. Note that the choice of the exact types of converters 1 and 2 remains a design issue.

To synthesize a desired impedance $Z(s)$, we have to ensure the correct magnitude and phase relationships between $v_{i}$ and $i_{i}$. Essentially we want $i_{i}$ to follow a reference template $i_{\text {ref }}$ which is related to $v_{i}$ as follows, in the complex frequency domain:

$$
I_{\text {ref }}(s)=V_{i}(s) G(s)
$$

where $G(s)$ is the transfer function from the input voltage to the reference template. Assume that the transfer function from $I_{\text {ref }}$ to $I_{i}$ is

$$
K(s)=\frac{I_{i}(s)}{I_{\mathrm{ref}}(s)} .
$$

Suppose $Z(s)$ is to be synthesized. Then, $G(s)$ becomes

$$
G(s)=\frac{1}{K(s) Z(s)} .
$$

Thus, in the circuit implementation, we have to realize this transfer function in order to synthesize the required impedance. A block diagram showing the control requirement is shown in Fig. 5. 


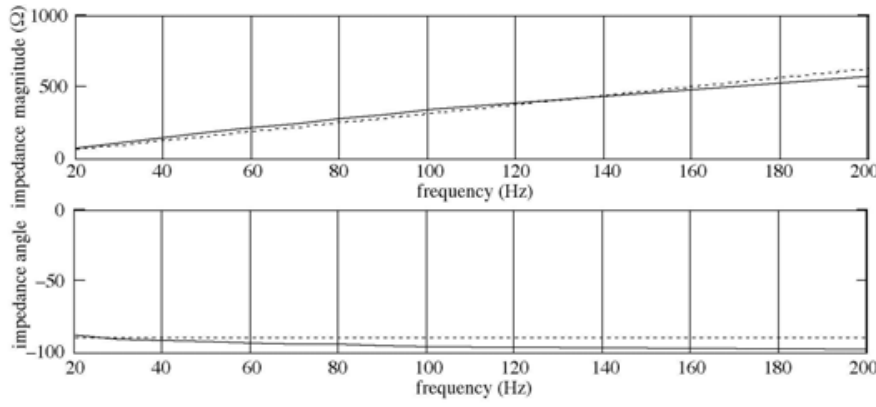

Fig. 9. Measured impedance magnitude and phase angle of the synthesized $0.5 \mathrm{H}$ pure inductance. Solid line: measured results from the experimental circuit; dashed line: ideal characteristic of an inductor of $0.503 \mathrm{H}$.

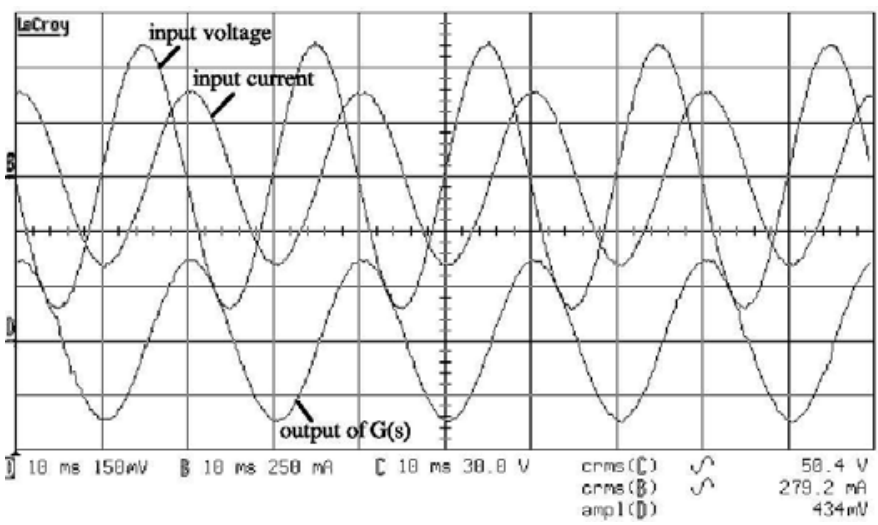

Fig. 10. Measured waveforms of synthesized pure inductance with input at $50 \mathrm{~Hz}$.

Clearly, $K(s)$ in general depends on the converter response. However, if the bandwidth of the converter response is much higher than that of the applied voltage $v_{i}$, we may assume that $K(s)$ is nearly a constant, i.e., $i_{i}$ is proportional to $i_{\text {ref }}$. Thus, we can find the control transfer function $G(s)$ from (5).

\section{Example 1: Pure Inductance Synthesis}

The impedance of a pure inductor is

$$
Z(s)=s L
$$

where $L$ is the inductance to be synthesized. Thus, the required $G(s)$ is

$$
G(s)=\frac{1}{s K L}
$$

which can be implemented using the circuit shown in Fig. 6. For other possible active circuit realizations, see [9]. With $R_{4}=R_{5}$ and $\left|s C_{1} R_{2}\right| \gg 1$, we have

$$
G(s) \approx \frac{1}{s C_{1} R_{1}} \Rightarrow L \approx \frac{C_{1} R_{1}}{K} .
$$

Hence, by appropriately choosing the values for $C_{1}, R_{1}$, $R_{2}$, etc., we can synthesize a pure inductance. It should be reiterated that the synthesis is effective only for the range of frequencies much lower than the switching frequency of the constituent dc/dc converters. For instance, choosing $C_{1}=$ $2.2 \mu \mathrm{F}, R_{1}=270 \mathrm{k} \Omega, R_{2}=2.2 \mathrm{M} \Omega, R_{4}=R_{5}=10 \mathrm{k} \Omega$, we
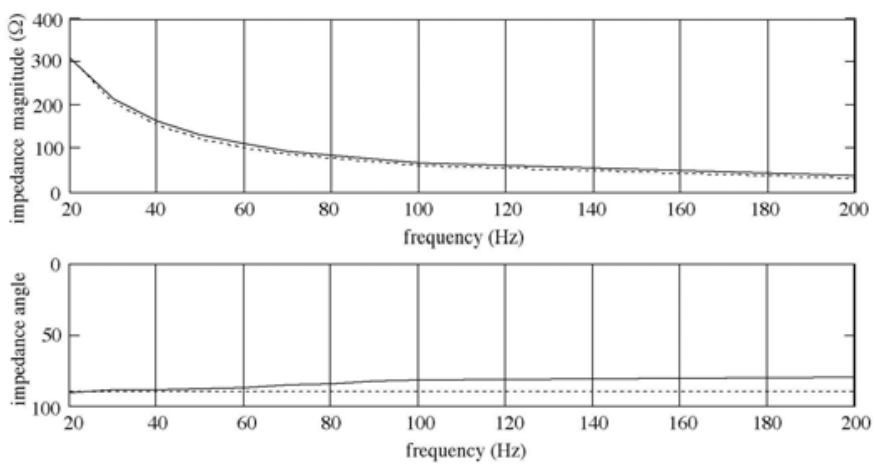

Fig. 11. Measured impedance magnitude and phase angle of the synthesized $25 \mu \mathrm{F}$ pure capacitance. Solid line: measured results from the experimental circuit; dashed line: ideal characteristic of a capacitor of $25 \mu \mathrm{F}$.

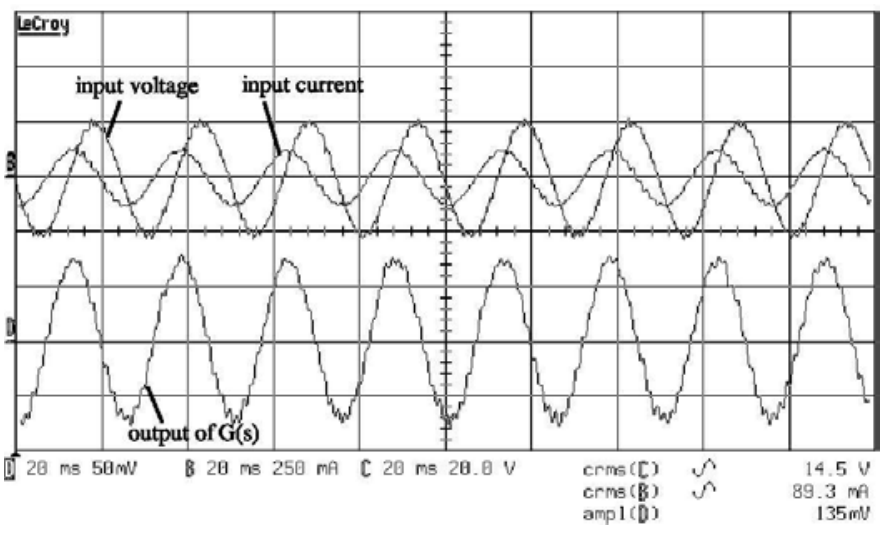

Fig. 12. Measured waveforms of synthesized pure capacitance with input at $50 \mathrm{~Hz}$.

have $\left|s C_{1} R_{2}\right| \gg 1$ for the frequency range $20 \mathrm{~Hz}$ to $200 \mathrm{~Hz}$. The synthesized inductance is $0.59 / K \mathrm{H}$, where $K$ can be predetermined experimentally.

\section{Example 2: Pure Capacitance Synthesis}

In a likewise manner, we may synthesize a pure capacitance. In this case, the required $G(s)$ is $s C / K$, if $C$ is the capacitance to be synthesized. The appropriate circuit for constructing $G(s)$ is shown in Fig. 7. With $R_{8}=R_{9}$ and $\left|s C_{2} R_{6}\right| \gg 1$, we have

$$
G(s) \approx s C_{2} R_{7} \quad \Rightarrow \quad C \approx K C_{2} R_{7}
$$

Hence, by appropriately choosing the values for $C_{2}, R_{6}$, $R_{7}$, etc., we can synthesize a pure capacitance. For instance, choosing $C_{2}=100 \mathrm{nF}, R_{6}=820 \mathrm{k} \Omega, R_{7}=220 \Omega$, $R_{8}=R_{9}=10 \mathrm{k} \Omega$, we have $\left|s C_{2} R_{6}\right| \gg 1$ for the frequency range from $20 \mathrm{~Hz}$ to $200 \mathrm{~Hz}$. The synthesized capacitance is $22 K \mu \mathrm{F}$, where $K$ can be found experimentally.

\section{Example 3: Inductive Impedance Synthesis}

The impedance of a series connection of an inductor $L$ and a resistor $R_{L}$ is

$$
Z_{L R}(s)=s L+R_{L}
$$



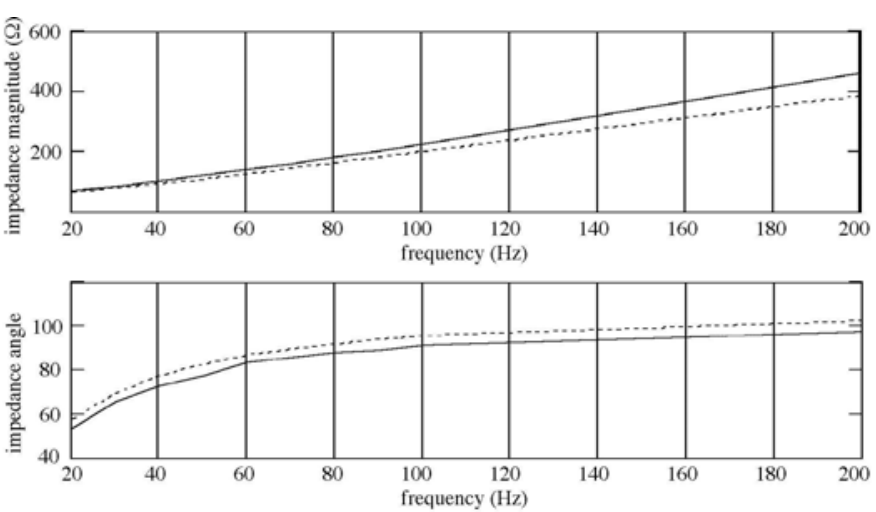

Fig. 13. Measured impedance magnitude and phase angle of the synthesized inductive impedance of of $50 \Omega$ in series with $0.3 \mathrm{H}$. Solid line: measured results from the experimental circuit; dashed line: ideal characteristic of the inductive impedance.

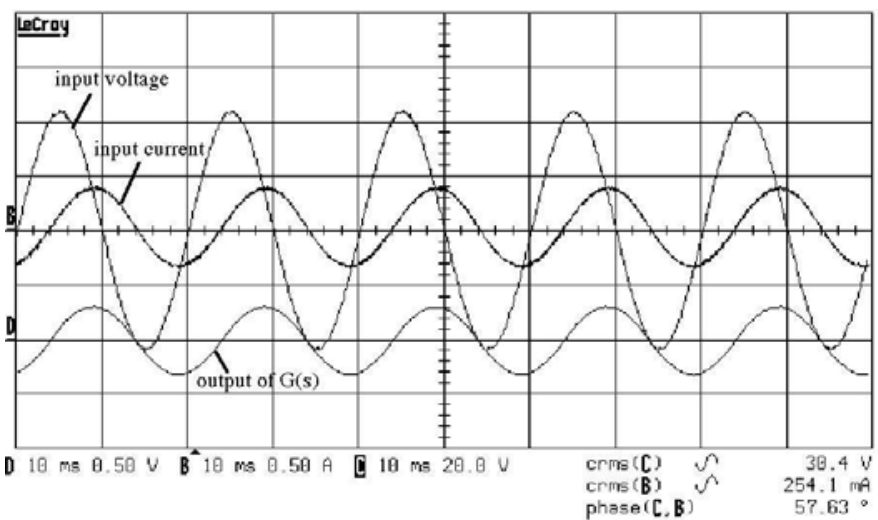

Fig. 14. Measured waveforms of synthesized inductive impedance with input at $50 \mathrm{~Hz}$.

and $G(s)$ can be calculated according to (5) as

$$
G(s)=\frac{1}{K R_{L}\left(1+\frac{s L}{R_{L}}\right)} .
$$

Thus, the transfer function $G(s)$ is a single pole system and can again be implemented with the circuit of Fig. 6 .

\section{Example 4: Capacitive Impedance Synthesis}

The impedance of a parallel connection of a capacitor $C$ and a resistor $R_{C}$ is

$$
Z_{C R}(s)=\frac{R_{C}}{1+s C R_{C}}
$$

and hence $G(s)$ can be calculated according to (5) as

$$
G(s)=\frac{1}{K\left(\frac{R_{C}}{1+s C R_{C}}\right)}=\frac{1}{K} \frac{1+s C R_{C}}{R_{C}} .
$$

Thus, the transfer function $G(s)$ is a one zero system and can also be readily implemented with the circuit of Fig. 7 .
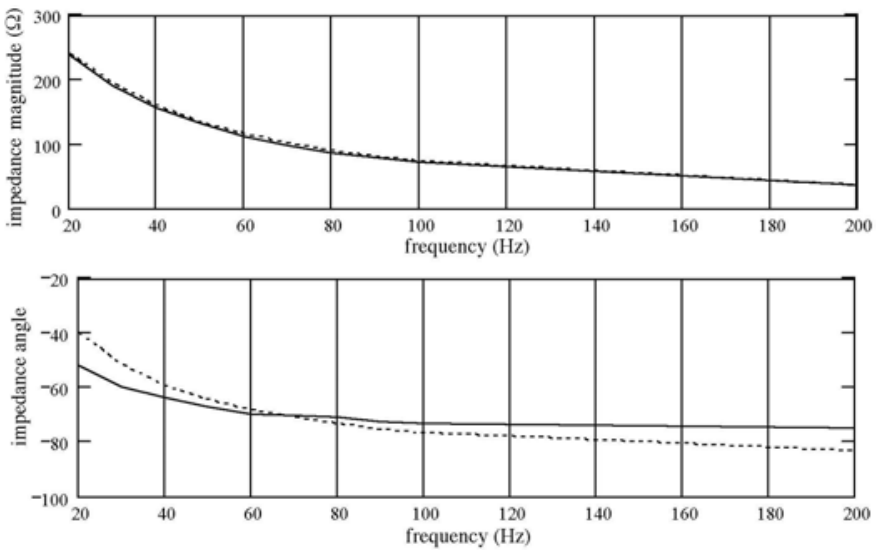

Fig. 15. Measured impedance magnitude and phase angle of the synthesized capacitive impedance of of $300 \Omega$ in parellel with $21 \mu \mathrm{F}$. Solid line: measured results from the experimental circuit; dashed line: ideal characteristic of the inductive impedance.

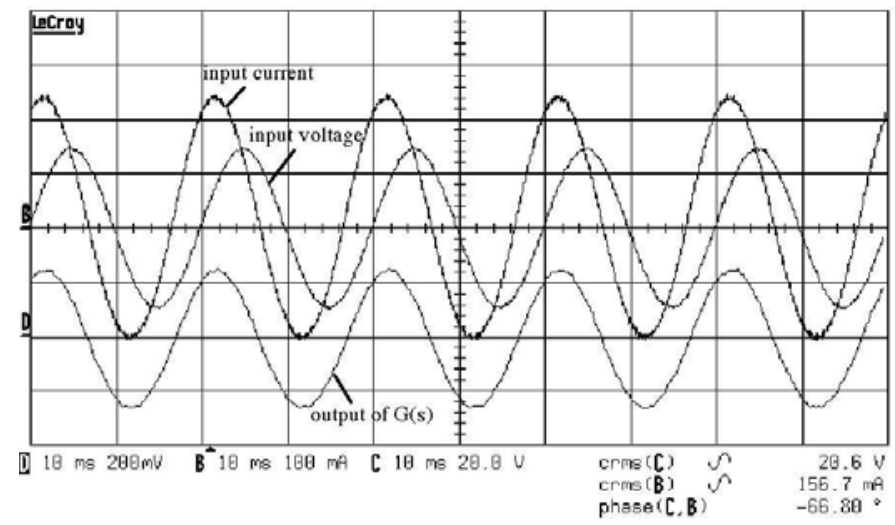

Fig. 16. Measured waveforms of synthesized capacitive impedance with input at $50 \mathrm{~Hz}$.

\section{EXPERIMENTAL RESULTS}

A particular choice of practical converter types for the implementation of the impedance synthesizer is shown in Fig. 8. Here, the input $v_{i}$ is a sinusoidal voltage source, and the output $v_{o}$ is connected to a current load. The switching frequency is $50 \mathrm{kHz}$. The four examples described in the previous section are evaluated.

Our first experiment is the synthesis of pure inductance. The inductance to be synthesized is $0.5 \mathrm{H}$. The circuit for realizing the $G(s)$ transfer characteristic has been shown earlier in Fig. 6 , and the parameters used are $C_{1}=2.2 \mu \mathrm{F}, R_{1}=$ $270 \mathrm{k} \Omega, R_{2}=2.2 \mathrm{M} \Omega$, and $R_{4}=R_{5}=10 \mathrm{k} \Omega$. The measured value for the gain $K$ is $1.18 \mathrm{~A} / \mathrm{V}$. As analyzed before, this set of parameters can provide a synthesized inductance of $0.59 / K=0.503 \mathrm{H}$ for the frequency range from $20 \mathrm{~Hz}$ to $200 \mathrm{~Hz}$. The test input voltage magnitude is $50.5 \mathrm{~V}$ (rms). The measured impedance magnitude and phase angle are shown in Fig. 9. A typical set of waveforms is shown in Fig. 10.

Our second experiment is the synthesis of pure capacitance. The capacitance to be synthesized is $25 \mu \mathrm{F}$. The circuit for realizing the $G(s)$ transfer characteristic has been shown 
earlier in Fig. 7, and the parameters used are $C_{2}=100 \mathrm{nF}$, $R_{6}=820 \mathrm{k} \Omega, R_{7}=220 \Omega, R_{8}=R_{9}=10 \mathrm{k} \Omega$. The measured value for the gain $K$ is $1.18 \mathrm{~A} / \mathrm{V}$. As analyzed before, this set of parameters can provide a synthesized capacitance of $22 K=25 \mu \mathrm{F}$ for the frequency range from $20 \mathrm{~Hz}$ to 200 $\mathrm{Hz}$. The test input voltage magnitude is $50.5 \mathrm{~V}$ (rms). The measured impedance magnitude and phase angle are shown in Fig. 11. A typical set of waveforms is shown in Fig. 12.

The third impedance to be synthesized is $L=0.3 \mathrm{H}$ in series with $R_{L}=50 \Omega$. We have realized $G(s)$ using the same circuit of Fig. 6, with $C_{2}=1 \mu \mathrm{F}, R_{1}=360 \mathrm{k} \Omega, R_{2}=6.2 \mathrm{k} \Omega$, and $R_{4}=R_{5}=10 \mathrm{k} \Omega$. The transfer function $K$ was found to be $1.18 \mathrm{~A} / \mathrm{V}$. This set of parameters gives a synthesized inductive impedance of $49.206 \Omega$ in series with $0.305 \mathrm{H}$, for the frequency range from $20 \mathrm{~Hz}$ to $200 \mathrm{~Hz}$. The test input voltage magnitude is $30.4 \mathrm{~V}$ (rms). The measured impedance magnitude and phase angle are shown in Fig. 13. A typical set of waveforms is shown in Fig. 14.

Our final impedance to be synthesized is a parallel connection of $C=21 \mu \mathrm{F}$ and $R_{C}=300 \Omega$. We have realized $G(s)$ using the same circuit of Fig. 7, with $C_{2}=10 \mathrm{nF}$, $R_{6}=660 \mathrm{k} \Omega, R_{7}=1.8 \mathrm{k} \Omega$, and $R_{8}=R_{9}=10 \mathrm{k} \Omega$. The transfer function $K$ was found to be $1.18 \mathrm{~A} / \mathrm{V}$. This set of parameters gives a synthesized capacitive impedance of $310.73 \Omega$ in parallel with $21.24 \mu \mathrm{F}$, for the frequency range from $20 \mathrm{~Hz}$ to $200 \mathrm{~Hz}$. The test input voltage magnitude is $20.6 \mathrm{~V}$ (rms). The measured impedance magnitude and phase angle are shown in Fig. 15. A typical set of waveforms is shown in Fig. 16.

\section{CONCLUSIONS}

A general impedance synthesis scheme using a minimum configuration of high frequency switching converters has been proposed in this paper. The method basically involve programming the input voltage to current relationship and providing the necessary power buffering capability. Using the proposed synthesis method, we can imitate any impedance for control purpose, such as in power factor correction. For the purpose of illustration, we have evaluated four cases experimentally, corresponding to synthesis of pure inductance, pure capacitance, lossy inductive impedance and lossy capacitive impedance.

\section{ACKNOWLEDGMENTS}

This work is supported by Hong Kong Research Grant Council under a CERG Grant (No. PolyU 5237/04E).

\section{REFERENCES}

[1] E. S. Kuh, "Special synthesis techniques for driving point impedance functions," IRE Trans. Circuit Theory, vol. 2, no. 4, pp. 302-308, Dec. 1955.

[2] A. Fialkow, "Inductance, capacitance networks terminated in resistance," IEEE Trans. Circuits Syst., vol. 26, no. 8, pp. 603-641, Aug. 1979.

[3] T. Nishi, "On the transformerless synthesis of reactance two-ports of degree four or less," IEEE Trans. Circ. Syst. I, vol. 15, no. 2, pp. 132-138, 1988.

[4] C.K. Tse, "Circuit theory of power factor correction in switching converters," Int. J. Circuit Theory Appl., vol. 31, no. 2, pp. 157-198, March 2003.

[5] O. Garcia, J. A. Cobos, R. Preito, P. Alou, and J. Uceda, "Single phase power factor correction: a survey," IEEE Trans. Power Electron., vol. 18, no. 3, pp. 749-755, May 2003.

[6] R. P. Severns and E. J. Bloom, Modern DC-to-DC Switchmode Power Converter Circuits, New York: Van Nostrand, 1985.

[7] N. K. Poon, C. K. Tse, and J. C. P. Liu, "Very fast transient voltage regulators based on load correction," IEEE Power Electron. Specialists Conf. Record, pp. 66-71, June 1999.

[8] C. P. Liu, C. K. Tse, N. K. Poon, M. H. Pong, and Y. M. Lai, "Synthesis of input-rectifierless ac/dc converters," IEEE Trans. Power Electron., vol. 19, no. 1, pp. 176-182, January 2004.

[9] R. Schaumann, M.S. Ghausi, and K.R. Laker, Design of Analog Filters: Passive, Active RC, and Switched Capacitor, Englewood Cliffs: Prentice Hall, 1990. 